\title{
Einüben von Geschlecht und Begehren. Plädoyer für eine rekonstruktive Sozialisations- theorie
}

Die Debatte um geschlechtsspezifische Sozialisation gehörte in den 1970er und 1980er Jahren zu den Ausgangspunkten der Auseinandersetzung um Geschlecht. Am Ende dieser beiden Dekaden löste sich die damalige Frauenforschung aus der zunächst sinnvollen engen Verklammerung mit dem Paradigma der Benachteiligung, die das Nachdenken über Sozialisation nachhaltig prägte, machte Geschlecht zur allgemeinen Analysekategorie und erarbeitete eine interdisziplinäre Herangehensweise. Doch veränderte sich der Stellenwert der Sozialisationstheorie nicht allein durch die Ausdifferenzierung in den Disziplinen, sondern ebenso durch Kritik aus den eigenen Reihen. Aus soziologischer Perspektive wurde schon früh gefordert, die den Individuen je eigene Weise der Aneignung von Zweigeschlechtlichkeit zu analysieren (Hagemann-White 1984). Anfang der 1990er Jahre kündigte sich eine endgültige Wende in den grundlegenden theoretischen Konzeptionen der sich fortan meistens der Geschlechterforschung zurechnenden Studien an, die Sozialisationstheorie gänzlich unzeitgemäß erschienen ließ. Unter den Vorzeichen von Identitätskritik, der Konzeption des Körpers als Inszenierung und dem Ringen um die Frage, wie individuelles Handeln und gesellschaftliche Struktur miteinander verschränkt seien, schienen Sozialisationstheorien ihren Schwerpunkt stets auf die Strukturen zu legen, Flexibilisierungen deshalb nicht fassen und den Körper in seiner Materialität nicht berücksichtigen zu können. Letzten Endes wurde diesen Theorien attestiert, dass ihnen ein Essentialismus nicht auszutreiben sei, sie die dichotome Geschlechterlogik unterstützten und letztlich ganz ad acta gelegt werden sollten.

Seit einigen Jahren wird dafür plädiert, dieses Moratorium zu beenden und den Faden der Sozialisationstheorie innerhalb der Geschlechterforschung unter veränderten Vorzeichen erneut aufzunehmen (Maihofer 2002, Bilden/Dausien 2006). Die Debatte um die Kritik an der Kategorie Geschlecht hat dafür, so eine These dieses Beitrags, das nötige Werkzeug bereitgelegt: erstens eine Fokussierung des Konstruktionsprozesses, also den Ansatz des doing gender und damit zunehmendes Interesse für Mikroanalysen; zweitens die Aufmerksamkeit für die Materialität des Körpers zwischen Flexibilität und Verfestigung und drittens die zunehmende Beachtung der Verschränkung von Geschlecht und Sexualität. Im Folgenden werden in einem ersten Schritt diese Schwerpunkte der Debatte rekapituliert, dann wird eine Fallstudie zu Geschlecht und Begehren in der ausge- 
henden Kindheit vorgestellt sowie auf Konstituenten einer Sozialisationstheorie hin untersucht, und auf dieser Grundlage wird aufbauend die Skizze einer rekonstruktiven Sozialisationstheorie vorgestellt.

\section{Rekonstruktionen unter dem Vorzeichen von Dekonstruktion}

Die Debatte um die Kritik an der Kategorie Geschlecht wird von Beginn an durch die Forderung begleitet, verstärkt empirische Forschungen durchzuführen (Landweer/Rumpf 1993). ${ }^{1}$ Mit dem Ansatz des doing gender wird die Herstellung von Geschlecht in konkreten Interaktionen lokalisiert und Geschlecht als fortlaufender Prozess bestimmt, welcher sich im Alltag in konkreten Darstellungen und Interpretationsleistungen zeigt. Geschlecht sei nicht immer schon da, sondern werde erst in seinem Vollzug in Körperhaltungen, dem Umgang mit kulturellen Objekten, in Ritualen, Alltagstheorien und Institutionen hervorgebracht und identifizierbar. In der Methodologie werden Zugänge ausgearbeitet, die zumindest annäherungsweise versuchen, Geschlecht als Kohäsionsidee einzuklammern und es $z \mathfrak{u}$ "sehen" und gleichzeitig nicht zu "sehen" (Hagemann-White 1993, Hirschauer 1993b, Gildemeister 2000, Kelle 2001).

In der sich im deutschsprachigen Raum etwa zeitgleich konstituierenden Kindheitsforschung wird die Teleologie eines Sozialisationsmodells kritisiert, die Kindheit stets in Hinblick auf das zu erreichende Erwachsensein konzipiert und Kinder auf diese Weise mit einem Defizit versieht; auch hier wird also - wenn auch nicht immer explizit - im Sinne einer Dekonstruktion von Dichotomien, die letztendlich als Hierarchien organisiert sind, vorgegangen. Nachdem Kinder, so die Argumentation, lange Zeit nicht als Akteure gesehen, sondern Kindheit psychologische Gesetzmäßigkeiten und Entwicklungsstadien zu illustrieren hatte, beschreibt die Kindheitsforschung Kinder als Produzenten von Kultur, deren Perspektiven herauszuarbeiten sind (z.B. Kelle/Breidenstein 1996, Honig/ Lange/Leu 1999). Statt der Untersuchung allgemeiner Entwicklungsprozesse werden Kindheiten in deren je unterschiedlichen Ausformungen anhand von Alltagspraxen häufig ethnographisch oder mit Hilfe von sprachzentrierten rekonstruktiven Methoden analysiert.

In Ansätzen der Jugendforschung, in denen Geschlecht zur zentralen Kategorie der Analyse gemacht wird, wird methodisch ebenso verfahren, doch kann die Jugend- im Unterschied zur Kindheitsforschung auf eine sehr viel längere rekonstruktive Forschungstradition zurückblicken, die nicht von Sozialisationsmo-

1 Nachdem in der ersten Phase der Frauenforschung Gesellschaftsanalysen in den Vordergrund gestellt wurden, waren Forschungen in den 1990er Jahren stärker an Mikroanalysen orientiert (Dausien/Kelle 2001). In den Sozialwissenschaften zeichnete sich allgemein ein Bedeutungszuwachs des qualitativen oder rekonstruktiven Paradigmas ab. 
dellen dominiert wurde. Die deutschsprachige Jugendforschung hat deutliche Impulse durch die britischen Cultural Studies erhalten, die Bezug nehmend auf frühe ethnographische Studien aus dem Umfeld des Symbolischen Interaktionismus eine Orientierung an rekonstruktiven Methoden bereits mitbrachten (Winter 2001).

Die Studien, die in der Folge an der Schnittstelle von Kindheit, Jugend und Geschlecht entstehen, stellen die Erwachsenenorientierung der Geschlechterforschung nachhaltig in Frage. Herausgearbeitet wird, entweder im Vergleich verschiedener Alterszeiträume oder in der Untersuchung eines solchen in vivo, auf welche Weise Kinder und Jugendliche ihr Aufwachsen gestalten (Breidenstein/ Kelle 1998, Breitenbach 2001, Budde 2005, Bütow 2005, Faulstich-Wieland 2004, Fritzsche 2003, Hackmann 2003, Jösting 2005, Tervooren 2006). ${ }^{2}$ Wenn die Genauigkeit dieser rekonstruktiven Studien in Rechnung gestellt wird, muss auch die Sozialisationstheorie ihre Reichweite präzisieren: Kinder und Jugendliche bringen ihr Alter ebenso wie ihr Geschlecht hervor, tun dies je nach Lebensaltern jedoch unterschiedlich. Theorien zu Sozialisation und Geschlecht müssen Alterszeiträume in den Fokus nehmen, in deren Rahmen dann die Requisiten, Handlungen und Situationen des doing age beobachtet und eine rekonstruktive Bestimmung der Stationen des Aufwachsens vorgelegt werden können. ${ }^{3}$

\section{Die allmähliche Verfestigung des Symbolischen in der Materie}

Die identitätskritische Haltung vor allem poststrukturalistischer Provenienz wird von Beginn an von der Warnung begleitet, den Körper nicht zu vergessen. Auch wenn die prominenteste Vertreterin Judith Butler gerade die Materialisierung der Körper zu theoretisieren trachtet, fordert die sprachtheoretische Fundierung ihrer Theorie den Vorwurf der Diskursontologie geradezu heraus (z.B. Landweer 1993). Wird sex im Unterschied zu gender lange Zeit auf der Seite der Natur im Sinne einer unveränderbaren Gegebenheit verortet, bestimmt Butler die Materie selbst als Schauplatz der Konstitution kultureller Formen (Butler 1995). Während sie das Konzept der Performance als Verknüpfung zwischen Körper und geschlechtlich codierten Positionen anbietet ${ }^{4}$, sieht Bourdieu, dessen Aufsatz

2 Die Frage, welche "biographische Hypothek diese Kindheit oder Jugend für das spätere Leben mit sich bringt, kann, so führt es Birgit Bütow aus, nicht beantwortet werden. Sie spricht sich deshalb - vor dem Hintergrund ihrer Studie zu Cliquen jugendlicher Mädchen - nachdrücklich für eine Trennung von Frauen- und Mädchenforschung aus (Bütow 2005, 247).

3 Der Übergang von der Kindheit zur Jugend ist dabei gut, die frühe Kindheit und erste Grundschulzeit fast gar nicht erforscht.

4 Wenn auch die Debatte um die Kategorie Geschlecht eine explizite Abgrenzung von Sozialisationstheorien vornahm, stellte sie dennoch den Begriff der "Inszenierung" und damit den Körper in den Mittelpunkt der Aufmerksamkeit, Begriffe, die nicht nur in der soziologischen Theorietradition des doing gender, sondern auch in Butlers »Performativität der Geschlechts- 
"Die männliche Herrschaft" (1997) große Beachtung innerhalb der Geschlechterforschung findet, mit seinem Konzept der "körperlichen Hexis" die Verbindung zwischen dem materiellen Körper und symbolischen Praxen als sehr viel dauerhafter an: Körperhaltungen, das Reden und das Gehen seien einverleibte "politische Mythologie" (Bourdieu 1999, Orig. 1980).

Auch Vertreterinnen und Vertreter der Kindheitsforschung legen ihren Überlegungen lange Zeit eine Trennung zwischen Natur und Kultur zugrunde, um Kinder vor einer Gleichsetzung mit Natur und einer durch sie applizierten Unmittelbarkeit und Unschuld in Schutz zu nehmen. Kindheit wird auf diese Weise jedoch allein als soziales und kulturelles Phänomen beschrieben, während die Materialität des Körpers von dieser kulturellen und sozialen Konstruktion ausgenommen bleibt (James/Jenks/Prout 1998, 146f.). Folgerichtig erhält die Materie des Körpers in ihrer Plastizität und Verfestigung in diesen Ansätzen zu wenig Aufmerksamkeit und wird die Verknüpfung zwischen der Ebene des Symbolischen und den materiellen Praxen weder empirisch noch konzeptionell ausgearbeitet. Erst in jüngster Zeit ist das Thema Körper, nicht zuletzt aufgrund dieser Versäumnisse, zu einem der zentralen Auseinandersetzungspunkte deutschsprachiger Kindheitsforschung geworden (Hengst/Kelle 2003).

Der Körper als Ort symbolischer Auseinandersetzungen ist in der Jugendforschung seit längerem eingeführt, denn in jugendlichen Szenen wird stets mit dessen Modellierung gearbeitet. So weisen Piercings, Tattoos, Accessoires, Kleidung etc. an der Oberfläche des Körpers Zugehörigkeiten aus und über Konsumpraktiken von Drogen oder der Askese etc. werden somatische Kulturen ausgebildet (King 2002, Kolip 1997). Eine Konzeption der allmählichen Verfestigung des Symbolischen in der Materie des Körpers, die von den konkreten körperlichen Praxen ausgeht und der Möglichkeit zur Veränderung von Normen in der Wiederholung Raum lässt, muss demnach Bestandteil einer aktuellen Sozialisationstheorie sein.

\section{Zwischen den Geschlechtern}

Innerhalb der Debatte um die Kritik an der Kategorie Geschlecht finden verstärkt Lebensformen von Geschlecht Beachtung, die das Feld dichotomer Geschlechterzuschreibungen überschreiten, verwirren oder in Frage stellen. Einer der Gründe liegt in der vermehrten Aufmerksamkeit für die Analyse von Sexua-

identität" einen zentralen Stellenwert einnehmen und, so meine Lesart, eine Vermittlung zwischen Handlung und Struktur vorzunehmen vermögen. Bezeichnet wird damit jeweils eine Inszenierung vor anderen, die für die erste Tradition einen sozialen Sinn in Interaktionen selbst herstellt und für die zweite Normen performiert und dabei - abgeleitet von der Figur der différance bei Derrida - immer einen Überschuss an Bedeutung produziert (Butler 1991). 
litäten, welcher nach und nach ein zentraler Platz innerhalb der Geschlechterforschung eingeräumt wird. So greifen Anfang der 1990er Jahre Stefan Hirschauer (1993a) und Gesa Lindemann (1993) Methode und Thema der ethnomethodologischen Studien Harold Garfinkels auf und legen empirische Untersuchungen zu Transsexualität vor. Identitätskritik, wie sie Judith Butler formuliert hat, führt queer als politischen und politisierenden Begriff in die Debatte ein und weist darauf hin, dass erst eine heterosexuelle Matrix Deckungsgleichheit zwischen sex, gender und Begehren erzwinge, jedoch sehr unterschiedliche Konstellationen dieser drei gelebt werden (Butler 1991, 1995). Wie diese Matrix wirksam wird, zeigt Butler auch an Beispielen aus dem Feld der Sozialisation, so z.B. die immer wieder thematisierte Anrufung nach der Geburt eines Kindes mit "It's a girl" oder "It's a boy", doch dienen diese eher der Illustration ihrer Theorie, als dass letztere sich durch die Rekonstruktion dieser Szenen in Frage stellen ließe. ${ }^{5}$

In jüngster Zeit hat darüber hinaus das Thema "Intersexualität" an Bedeutung gewonnen. Im Fokus der Debatte steht eine Kritik an Normalisierungen des Körpers in der Kindheit, der, ist das Geburtsgeschlecht nicht dem einen oder dem anderen Geschlecht zuzuordnen, konkret durch medizinische Operationen, aber auch durch das Einüben eindeutiger Geschlechterinszenierungen im Prozess von Erziehung und Sozialisation geformt wird (Butler 2002, Dietze 2003, Klöppel 2002, NGBK 2005, Lang 2006). ${ }^{6}$ Die Aufmerksamkeit für Kindheit und Jugend ist diesem Projekt stets eingeschrieben, da die zentralen Weichenstellungen bereits kurz nach der Geburt häufig medizinisch angebahnt und auf der Ebene der Erziehung mit dem Ziel einer eindeutigen Geschlechterdarstellung fortgesetzt werden.

Vor dem Hintergrund dieser Theorieentwicklungen wird in rekonstruktiven Studien zu Kindheit und Jugend Sexualität als Aneignungsprozess analysierbar, in der die gleichgeschlechtliche Peer-Group eine zentrale Rolle spielt, weil diese gleichzeitig Ort der Inszenierung ist und Akteure für die Inszenierung bereitstellt (Breidenstein/Kelle 1998, Breitenbach/Kausträter 1999, Fritzsche 2003, Jösting 2006, Tervooren 2006). Das kann jedoch nur gelingen, wenn nicht nach kohärenten Identitäten gesucht wird, sondern die einander durchkreuzenden, sich gegenseitig verstärkenden oder in Frage stellenden Begehrenslinien innerhalb der gleich- und gemischtgeschlechtlichen Peer-Groups aufgesucht und rekonstruiert werden.

5 Im US-amerikanischen Diskurs ist die Schnittmenge zwischen Queer Theory und Kindheitsforschung größer (Bruhm/Hurley 2004, Halberstam 1999, Rottnek 1999, Zevy 1999), im deutschsprachigen Raum finden sich mehrheitlich solche Studien, die sich mit Erwachsenen beschäftigen (Hartmann 2004, Hartmann et al. 2007, Hänsch 2003) und nur in wenigen empirischen Studien zu Kindheit und Jugend wird dieser Ansatz eingearbeitet (Hackmann 2003, Fritzsche 2003, Tervooren 2004, 2006).

6 Die historische Untersuchung Foucaults zu Herculine Barbin liegt zwar schon seit langem vor, hat aber im Zuge der aktuellen Beschäftigung mit dem Phänomen an Bedeutung gewonnen (Foucault ${ }^{3}$ 1998). 


\section{Zur Jugendlichen werden}

Die ethnographische Studie ${ }^{7}$, aus der im Folgenden ein Beispiel vorgestellt wird, fußt auf einer Untersuchung zehn- bis dreizehnjähriger Kinder in einer sechsjährigen Berliner Grundschule. Auf der Grundlage von teilnehmender Beobachtung, Videobeobachtung, Gruppendiskussionen und Videoinszenierungen, in denen vor allem die Aktivitäten der Kinder untereinander in den Blick genommen wurden, werden in der Auswertung des Materials die Merkmale des Prozesses des Zum-Geschlecht-Werdens dieser Kinder systematisch zusammengestellt. Methodologisch wird die Optik der Zweigeschlechtlichkeit auf der Grundlage einer sozialwissenschaftlichen Adaption des Prinzips der "Nachträglichkeit « ${ }^{8}$ verfremdet. Um der Frage nach den konkreten Inszenierungen von Geschlecht, Körper und Begehren am Ende der Kindheit nachzugehen, wird die Schlüsselszene des von einer Clique elf- bis dreizehnjähriger Mädchen selbst entworfenen, gedrehten und gespielten Videofilms vorgestellt. ${ }^{9}$ Das Beispiel wird vor dem Hintergrund von drei Perspektiven analysiert, die in der Sozialisation am Übergang von Kindheit und Jugend zentral sind: Die Rolle der Rituale im Prozess des Aufwachsens, die Bedeutung der Peer-Group und die Stilisierung jugendlicher Posen.

Der Film erzählt die missglückende Initiation des Mädchens Chrizzy in eine Mädchenclique. Eine der Wortführerinnen der realen Clique fasst die Situation folgendermaßen zusammen: "Wir nutzen sie dann halt aus, wir spannen ihr den Freund aus; also ständig, dass sie uns was spendiert beispielsweise, also, sie hat halt mehr Geld als wir. Und irgendeines Tages, wo sie dann kein Geld mehr hat, dann schmeißen wir sie raus." Die Novizin zeigt von Anfang an, dass sie bereit ist, alles für die Clique aufzugeben. Zunächst verleugnet sie ihre beste Freundin. Als die beiden Wortführerinnen der Mädchenclique zufällig erfahren, dass sie mit Mike, einem in der Schule beliebten Jungen liiert ist, beschließen sie, ihr Mike mauszu-

7 Die Studie ist in dem Projekt "Die Hervorbringung des Sozialen in Ritualen und Ritualisierungen" im Sonderforschungsbereich »Kulturen des Performativen" (FU Berlin) entstanden.

8 Der Begriff der "Nachträglichkeit» wird hierbei der Filmtheoretikerin Kaja Silverman entliehen und ist furr die Auswertung vor allem von Videobeobachtungen fruchtbar gemacht worden. Silverman geht davon aus, dass zu einem bestimmten Zeitpunkt in einer Gesellschaft ein Bildrepertoire vorhanden ist, von dem sich zu distanzieren nur durch einen zeitlichen Abstand vom Sehen oder seiner technischen Fixierung möglich wird. Ausführlich werden diese methodologischen Grundlagen an anderer Stelle ausgeführt (Tervooren 2006, 56ff.).

9 Die Themen und deren Bearbeitung, die sich in diesem fiktiven Material zeigen, lassen sich auch in den teilnehmenden und videogestützten Beobachtungen des Alltags dieser Mädchen in der Schule und in der mit ihnen durchgefuihrten Gruppendiskussion auffinden. Meines Erachtens ermöglicht der Film einen Zugang zu den Phantasien der Mädchen über ihr Aufwachsen und diese sind ebenso Bestandteil einer Geschlechtersozialisation wie die konkreten Handlungen. Die Figuren im Film sind etwa zwei Jahre älter als die realen Mädchen. In der ethnographischen Studie zeigen sich die Machtstrukturen der Gruppe, das "Nachmach-Verbot", die engen Bindungen unter den Mädchen und Prozesse der Exklusion jedoch auch in den Alltagshandlungen der Kinder. Der Film setzt vor allem die größte Angst, die das Jugendlichwerden provoziert, in Szene, die Angst davor, nicht dazugehören zu dürfen. 
treiben", um ihr eine Lektion zu erteilen. Sie geben Chrizzy den dringlichen Rat, sich von ihrem Freund zu trennen, da er, so ihre Behauptung, einen schlechten Charakter habe. Die Initiandin gibt Mike auf der gemeinsam mit der Clique besuchten Fete kurz und knapp den Laufpass. Der abservierte Mike zeigt keine Regung und fragt die unmittelbar nebenan sitzenden Mädchen der Clique, ob eine der drei mit ihm tanzen wolle und die Cliquenchefin tanzt mit ihm. Chrizzy, die jetzt begreift, dass sie gezielt öffentlich bloßgestellt wurde, muss sich noch anhören: "Du kannst nie eine von uns werden!«, und ihr kurzer Traum von Zugehörigkeit wird endgültig zum Alptraum.

\section{Wiederholen und verändern: Das Ritual}

Die Novizin ist bestrebt, den Übergang in die Adoleszenz durch ein Angliederungsritual zu meistern. ${ }^{10}$ Die Mädchenclique, in der sich Chrizzy durch ihren übergroßen Eifer von vorneherein disqualifiziert, fingiert das Initiationsritual jedoch. Beim ersten gemeinsamen Fetenbesuch der Novizin mit der Clique wird dieser gezeigt, dass sie aufgrund ihrer großen Beflissenheit eben nicht dazugehören darf. Als Königsweg in die Adoleszenz wird der Prozess der Individualisierung aufgefasst und "nicht nachmachen" ist das zentrale Gebot dieser Clique. Damit hat die Novizin die Fallen des Paradoxons "Werde wie wir und werde nicht wie wir" verkannt und muss, nachdem sie bereits die ersten beiden Phasen des Rituals hinter sich gebracht zu haben scheint, auf Zugehörigkeit verzichten.

Sozialisationstheorie hat einerseits die Tradierung des Überlieferten und andererseits deren Veränderung im historischen Prozess und in der individuellen oder gruppenspezifischen Aufführung durch die Akteure zu erklären. Da das Moment der Wiederholung kennzeichnend für Rituale ist, bieten diese nicht nur den günstigsten Ansatzpunkt zur Beschreibung von Kulturen, so die These, sondern auch für den Prozess der Sozialisation. Weil sie nicht spontan stattfinden und auf ihre Wiederholung Verlass ist, ist in ihnen die Möglichkeit des Erprobens und Veränderns gegeben. Rituale gelten in Erziehung und Sozialisation als Prozesse der Identifizierung und Transformation, in welchen neue Identitäten gebildet und Menschen zu Mitgliedern von Gruppen werden, und verweisen so auf die Tradierung von etwas, was es bereits gibt (Wulf et al. 2001, Wulf/Zirfas 2004).

In Mikroanalysen zu Geschlecht, Kindheit und Jugend gerät zunächst das Subjekt als Handelndes, das seinen Körper und sein Begehren inszeniert, in den

10 Gerade das Ende der Kindheit wird in vielen Kulturen durch Rituale begleitet, die einen Übergang in die Adoleszenz choreographieren. Heute lässt sich eine Tendenz zur Informalisierung von Ritualen ausmachen: wenn Rituale veranstaltet werden, nehmen die Heranwachsenden diese häufig selbst in die Hand. Die Entlastung, die Rituale in vormodernen Zeiten verschafften, kann auf diese Weise nicht mehr in Anspruch genommen werden. 
Mittelpunkt der Aufmerksamkeit. Damit bleibt aber weiterhin die Frage offen, wie eine Vermittlung zwischen dem "bottom-up-Prozess" der Rekonstruktion von Handlungspraxen und dem "top-down-Prozess" eines sozialisationstheoretischen Entwurfes entwickelt werden kann. ${ }^{11} \mathrm{Um}$ an der Schnittstelle zwischen Rekonstruktion und Sozialisationstheorie zu arbeiten, werden Rituale methodisch gewendet und als Ansatzpunkte von Beobachtung und Analyse bestimmt. Durch das Moment der Wiederholung vermitteln sie zwischen den beiden prototypischen Beschreibungsweisen von Kindheit und Jugend. Über die synchrone empirische Beschreibung der Gleichaltrigenkultur hinaus wird durch die Rituale einer diachronen Dimension des Aufwachsens, wie sie die Sozialisationstheorie vorschlägt, zumindest Rechnung getragen. Darüber hinaus können mit dem Fokus auf den Ritualen einerseits mit Bedeutung aufgeladene Situationen aus dem Material herausgearbeitet werden und andererseits deren Vollzug in konkreten Praktiken beschrieben werden und damit konkrete Aussagen über Praktiken, die Lebensalter konstituieren, gemacht werden. So können Ausschnitte lebenslanger Sozialisation rekonstruiert und die Geltungskraft der Theorie präzisiert werden.

\section{Erproben und Zeigen: Die Peer-Group}

Die Schlüsselszene des Films ist durch einen Jungentausch ${ }^{12}$ zwischen dem neuen Mädchen und den Mädchen der Clique strukturiert. Die Novizin opfert ihren Freund Mike der Clique und erwartet als Gegengabe, dass ihre Zugehörigkeit auf Dauer bestätigt wird. Diese antizipierte Gegenseitigkeit lösen die Mädchen der Clique jedoch nicht ein. Indem sie den Jungen zum konkreten Tauschobjekt machen und ihn als Trophäe für den Sieg der Mädchenclique über Chrizzy präsentieren, verweigern ihr die Akteure der Clique nicht nur die Aufnahme, sondern führen vor, dass das Konzept, alles für sie zu opfern, scheitern muss.

Hatte Chrizzy sich ausgemalt, die Cliquenchefin könne zu ihrer Freundin werden, wird diese stattdessen zu ihrer Rivalin, die öffentlich mit ihrem Freund tanzt, um sie zu demütigen. Die Chefin der Clique folgt dabei nicht ihrem eigenen Begehren, sondern dem der ausgeschlossenen Chrizzy, die damit zur Mittlerin wird. In der Rivalität wird dieVerbindung zwischen beiden also nicht durch-

11 Auch das Themenheft "Rekonstruktive Geschlechterforschung" der Feministischen Studien (2001) legte zwar gegenstandstheoretische Analysen von Geschlecht vor, berücksichtigte sozialisationstheoretische Überlegungen jedoch nicht.

12 Der Begriff des Jungentauschs spielt auf den Frauentausch an, den der Anthropologe LéviStrauss in "Die elementaren Strukturen der Verwandtschaft" (1993, Orig. 1949) beschreibt. Der Tausch der Frauen unter den Männern festigt die Verwandtschaftsstrukturen der Männer, während die Frauen in diesem Arrangement die Identitäten der Männer spiegeln. 
trennt, sondern in einer modifizierten Weise weitergeführt, ein Arrangement, das der Anthropologe René Girard als "trianguläres Begehren" bezeichnet. Girard spricht vom Begehren gemäß dem Anderen, das eine intensive machtvolle Verbindung unter den Begehrenden schafft, da das Begehren eines anderen zum ureigensten Begehren transformiert wird (Girard 1999, Orig. 1961, 13f.). In diesem Jungentausch verlaufen die Linien der Triangel also von Chrizzy und der Mädchenclique zu dem Jungen Mike und, da alle Mädchen das gleiche Objekt des Begehrens favorisieren, auch zwischen den beteiligten rivalisierenden Mädchen.

Die Pointe dieses Beispiels ist jedoch, dass keine tatsächliche Rivalität um den Jungen besteht. Dieser ist sowohl für Chrizzy als auch für die anderen Mädchen austauschbar und wird regelrecht weitergereicht. Der Wunsch der Initiandin nach Bindung an die Mädchen der Clique ist ungleich größer als der Wunsch, sich an den Jungen zu binden. Auch für die Mitglieder der Mädchenclique ist der einzige Junge, der im Film überhaupt eine Rolle spielt, nicht als einzelner, sondern als Typus von Bedeutung. Für sie hat Mike vor allem die Funktion, die Grenzen ihrer Clique zu markieren. Nach außen grenzen sie mit seiner Hilfe Chrizzy aus und nach innen benutzen sie ihn, um Positionen im Feld der Heterosexualität einzuüben. Der Austausch der Affekte vollzieht sich vor allem zwischen allen beteiligten Mädchen, während der Junge in dieser Ökonomie zum Objekt wird, an dem Wünsche und Begehren der Mädchen verhandelt und vorgeführt werden. So durchkreuzen sich in dieser zentralen Szene mehrere Energien: das Begehren einer Neuen, zur Clique zu gehören, die Verbindung unter den Mädchen sowie die Auffuihrung heterosexuellen Begehrens in der Öffentlichkeit. ${ }^{13}$

Gleichaltrige stellen ein wichtiges Feld des Erprobens angemessener Geschlechterinszenierungen zur Verfügung. Kulturelles Wissen wird dabei nicht allein vermittelt, sondern über einen langen Zeitraum ausprobiert und in Inszenierungen, aber auch in handfesten verbalen Auseinandersetzungen verarbeitet. In solchen Prozessen entsteht eine präzise Vorstellung von der richtigen und falschen Inszenierung, die, verlockt durch den Wunsch nach Zugehörigkeit, die Peers dazu anreizt, diesen Inszenierungs- und Verkörperungsformen zu folgen, da nur so der eigene Aufstieg in der hierarchisch strukturierten Gruppe zu organisieren ist. Die an Vorbildern orientierten oder auch zufällig entstandenen Entwürfe Einzelner oder Mehrerer werden gemeinsam wiederaufgeführt und kön-

${ }_{13}$ Eve Kosofsky Sedgwick, die mit der Triangel nach Girard arbeitet, schlägt vor, diese unterschiedlichen Energien analytisch nicht als Gegensatzpaar auszuweisen, sondern auf einem Kontinuum der Begehrensformen anzusiedeln. Für Weiblichkeit wurde solch ein Kontinuum immer schon zugrunde gelegt, während bei Bindungen zwischen Männern zwischen sexuellen und allein sozialen Beziehungen getrennt wird. Sedgwick spricht von "homosozialem Begehren", um die Entgegensetzung von Identifizierung und Begehren, wie sie im Freudschen ödipalen Dreieck entwickelt wurde, zu entkräften (Sedgwick 1985). 
nen in dieser spezifischen Form kollektiver Wiederholung kritisiert, verworfen oder weiterentwickelt werden. Was zunächst als Verfehlen kohärenter Geschlechtsidentitäten erscheinen konnte, wird zur Erprobung unterschiedlicher Geschlechterinszenierungen von Kindern in der angehenden Adoleszenz. Zentral für diesen Prozess ist das Inszenieren für andere als ein Zeigen des eigenen Körpers.

So trachten Kinder in der ausgehenden Kindheit in ihrem Begehren weniger nach Erfüllung, als dass es die Beteiligten zunächst für sich und für andere aufführen. Für das Ende der Kindheit wird Begehren deshalb als ein drängendes Verlangen, zueinander zu gehören, definiert, das Körperlichkeit oder die Imagination erster sexueller Akte einschließen kann, jedoch nicht muss. Der Begriff beschreibt die Übergänge zwischen sexuellem und sozialem Begehren, die Beziehung zur besten Freundin ebenso wie das erste "Zusammensein" mit dem Freund oder der Freundin. Begehren wird also stets auf ein oder mehrere gegenoder gleichgeschlechtliche menschliche Gegenüber bezogen. Anhand des empirischen Materials wird Begehren als ereignishafte, ständige Auffuihrung aufgefasst, die sich nach und nach in Begehrensformen verfestigt. Ausdrucksmuster, Aufführungsformen und Praktiken von Geschlecht in unterschiedlichen Beziehungskonstellationen werden auf diese Weise nicht schon von vornherein auf einen "hinter" diesen Formen liegenden Sexualtrieb zurückführt. Das analytische Vorgehen, welches die Performanz des Begehrens untersucht, setzt vielmehr umgekehrt bei den Praktiken selbst an und entwickelt von ihnen ausgehend eine Deutung, weshalb von Begehrenslinien gesprochen wird.

Begehrensformationen in ihrer Verbindung mit Geschlechterperformanzen und die Vorteile verschiedener Inszenierungen können erprobt ebenso wie die Verluste erfahren werden, die sich einstellen, wenn man z.B. nicht mit der Person des anderen Geschlechts gesehen wird. Bei den Kindern ist dieses jedoch zunächst nicht mehr als das rituelle Sich-Zeigen im Rahmen von Peer-Groups und darüber hinaus. Inszenierungen von Geschlecht und Begehren sind tentativ und deshalb sind die angehenden Jugendlichen auf ein Publikum angewiesen, das Experimentierfeld, Vorbild und Preisrichter in einem ist. Durch die Aufmerksamkeit für die komplexe Verbindung von Geschlecht und Begehren konnten Besonderheiten der ausgehenden Kindheit erarbeitet und die Mädchenclique als Raum beschrieben werden, an dem sich unterschiedliche gleich- und gegengeschlechtliche Begehrenslinien treffen (Tervooren 2006). Den Aktivitäten der Mädchen wird somit kein Ziel unterstellt und die Clique gerade nicht als Ort der Vorbereitung auf ein späteres heterosexuelles Erwachsenenleben charakterisiert. 


\section{Stilisieren und Verkörpern: der öffentliche Tanz}

Auffallend ist, dass die Cliquenchefin stellvertretend für die anderen Mädchen mit dem Jungen vor einem und für ein Publikum tanzt. Ein Mädchen aus der Clique darf dies, muss es aber auch tun, um den Sieg der Clique und damit den endgültigen Ausschluss Chrizzys zu demonstrieren und die Grenzen der Clique dauerhaft zu bestätigen. Die Inszenierung des Paartanzes - mit Aufforderung durch den Herrn - verlangt eine heterosexuelle Inszenierung und darüber hinaus eine individuelle Performance. Ein Mädchen muss sich aus der Clique herauslösen, der Neuen und allen anderen zeigen, dass diese nicht dazugehört, und diese Rolle übernimmt nach kurzer Rückversicherung mit den anderen die "Chefin" der Clique. Sie tanzt für die anderen mit und durch ihre Stellvertretung haben alle Cliquenmitglieder Anteil an der Inszenierung. Das Modell der (romantischen) Liebe zwischen zweien widerspricht der Erlebniswelt der Kinder. Sie lösen diesen Widerspruch, indem die Mächtigste unter ihnen stellvertretend für die anderen tanzt. Damit führt sie ein heterosexuelles Begehren auf, das aber den Belangen der gleichgeschlechtlichen Clique nachgeordnet bleibt.

Im Alter zwischen zehn und dreizehn Jahren schicken die Kinder sich an, jugendliche Geschlechterinszenierungen einzuüben. Angehende Jugendliche müssen, um ihre Altersangemessenheit zu sichern, die eigenen Geschlechterinszenierungen grundlegend verändern. Kinder experimentieren mit den ihnen zugänglichen Inszenierungen der Erwachsenen. Umgekehrt wird ihnen von Gleichaltrigen und Erwachsenen eine zunehmend auf die eigene Sexualität ausgerichtete Geschlechterinszenierung unterstellt. Eine angemessene Inszenierung wird durch Stilisierungen erprobt, die allmählich in das selbstverständliche Repertoire des Körpers übergehen und nach und nach zu einem körperlichen Stil werden.

Bereits die angemessene Geschlechterinszenierung verspricht in diesem Alter des Übergangs Rechte, die zunächst noch Jugendlichen und Erwachsenen vorbehalten sind. Diese Rechte sind mit der Verantwortung für einen korrekten Umgang mit den Ressourcen des Geschlechts verbunden. So können »die kulturellen Genitalien als ultimative Begründung für die Legitimität von Geschlechterdarstellungen und Lebensweisen verliehen [werden] (Hirschauer 1993, 51). Die körperlich sichtbaren Veränderungen wie das Wachstum der sekundären Geschlechtsmerkmale gehen dem Einüben voraus, verlaufen parallel zu ihnen oder folgen nach ihnen. Steht am Ende der Kindheit zunächst die Arbeit an der überzeugenden Stilisierung eines jugendlichen Geschlechts im Vordergrund, die sich nach und nach in einen körperlichen Stil wandelt, markieren mit zunehmendem Alter auch die körperlichen Veränderungen die Ankunft in der Adoleszenz.

Kinder, aber auch Jugendliche, üben also die historisch sich wandelnden Geschlechterverhältnisse in ihren Geschlechterinszenierungen ein und setzen darüber gleichzeitig Veränderungen derselben in Gang. Geschlecht ist ein praktisches Wissen über seine Inszenierung, das von Generation zu Generation weitergege- 
ben und von klein auf eingeübt wird. "Verkörperung" wird deshalb als kultureller Akt konturiert und als das allmähliche Verfestigen der Geschlechterinszenierungen in der Materialität des Körpers umschrieben. Sie ist sowohl auf der Ebene des Symbolischen als auch auf der des Materiellen angesiedelt. Die Verkörperung ist zwischen der Inkorporierung von Machstrukturen, dem zitatförmigen Einzelfall und dem Erproben der Kinder im kollektiven Rahmen der Peer-Group angesiedelt. Wird die Sozialisationstheorie wieder aufgegriffen und auf dem Feld des Körpers ausbuchstabiert, kann über den Inszenierungsbegriff die Plastizität der Materie und über den Begriff der Verkörperung die Verfestigung des Symbolischen in der Materie, wie sie eine Lebensgeschichte mit sich bringt, berücksichtigt werden.

\section{Einüben von Geschlecht und Begehren: Skizze einer rekonstruktiven Sozialisationstheorie für die ausgehende Kindheit}

Der Dreischritt von "wiederholen und verändern", "erproben und zeigen* und "stilisieren und verkörpern", wie er für die ausgehende Kindheit konturiert worden ist und der nur analytisch als ein sukzessiver Prozess beschrieben werden kann, tatsächlich aber meistens gleichzeitig geschieht, soll im Folgenden "Einüben von Geschlecht und Begehren" genannt werden. Der Prozess des Einübens ${ }^{14}$ meint die Wiederholung konkreter Handlungen, die fehlgehen, korrigiert und erneut inszeniert werden können. Das Präfix "ein" unterstreicht die Wiederholung des Übens und verweist auf die gesellschaftlichen Strukturen, die von den Kindern aufgegriffen werden. Das Verb "üben" bezieht immer schon das Fehlgehen mit ein, ohne die Bedeutung des "Noch-nicht-Könnens" negativ zu konnotieren. "Einüben" betont die Unabdingbarkeit der "Verfehlung" und beschreibt damit ihr produktives Potential, das in der Neuschöpfung liegt. In der notwendigen Verfehlung der Bilder oder Vorbilder entsteht etwas Neues, das es so zuvor nie gegeben hat.

Im Einüben vollzieht sich der Bezug auf das Vorangegangene ${ }^{15}$ in erster Linie mimetisch, wiederholt etwas und verändert es zugleich. Transformationen schließen den Bezug auf das Vorangegangene immer schon mit ein: Im mimetischen Noch-einmal-Machen entsteht etwas, was es zuvor so noch nicht gegeben hatte (Gebauer/Wulf 1998). Da Geschlechterverhältnisse und Geschlechterinszenierungen historisch bedingt und stets veränderlich sind, bilden sich neue

14 "Einüben" ist in Anlehnung an die "Strukturübungen" von Pierre Bourdieu entwickelt worden (1999, Orig. 1980, 138), betont aber weniger den Gedanken der Struktur, als den des Übens (ausführlicher Tervooren 2006, 21ff.).

15 Den Begriff des "Vorangegangenen" verwende ich, um Normen, Werte und ästhetische Formen in Bezug auf ihre Tradierung zu kennzeichnen. Der Begriff "Norm" allein, wie ihn etwa Butler benutzt, berücksichtigt die ästhetische Dimension des Sozialen nicht ausreichend. 
Muster nicht, weil die Kinder ein Vorbild verfehlen, sondern weil sie neue, dem Alter der Kinder oder Jugendlichen angemessene und den Modernisierungsprozessen unterworfene Formen von Geschlecht und Begehren erproben. Möglichkeiten des Erprobens vollziehen sich am Ende der Kindheit in ritualisierter Art und Weise, häufig im Rahmen einer Peer-Öffentlichkeit. In der Gruppe der Gleichaltrigen werden Geschlechterinszenierungen ausprobiert, gezeigt, angenommen oder verworfen und erneut inszeniert. Sie wird so zur Instanz der Bewertung angemessener und unangemessener Inszenierungen von Geschlecht und Begehren und modelliert den Prozess des Einübens.

Im Einüben entstehen dann Stilisierungen von Geschlecht, denen die Arbeit, welche die Kinder in sie investieren, noch sehr anzumerken ist. Avisierte Körperstile, Formationen von Macht und Geschlecht und Inszenierungen von Begehren werden in der Stilisierung wiederholt und erprobt. Diese Stilisierungen können, müssen aber nicht auf den körperlichen Veränderungen, welche die Adoleszenz mit sich bringt, fußen; viele Kinder nehmen diese in der Inszenierung vorweg und produzieren einen zu diesem Zeitpunkt noch fiktionalen Körper. Sie erweitern den eigenen Körper in ihren Körperstilen prothetisch und evozieren eine Gestalt, die sie noch nicht besitzen. In der beständigen Wiederholung der Geschlechterinszenierungen durch die Peer-Group werden diese nach und nach verfestigt. In der Materie verkörpern sich die symbolischen Formen und ihre Macht im Körperstil. Dieser Prozess unterliegt beständigen Korrekturen durch die Peer-Group, hinterlässt jedoch Spuren in der Materie.

Damit ist Einüben sowohl durch die Eigenaktivität des Subjekts als auch durch das Geschehen und Geschehenlassen charakterisiert. Einüben umschreibt einen Prozess, in dem bereits existierende Inszenierungen von Geschlecht und Begehren aufgegriffen und verändert wiederaufgeführt werden, der Möglichkeiten des Erprobens einbezieht und von der ereignishaften Inszenierung zur Verkörperung von Geschlecht und Begehren führt. Am Ende der Kindheit muss die eigene Geschlechterinszenierung an vielen Stellen verändert werden, so dass das Einüben sich durch die zahlreichen Stilisierungen überdeutlich zeigt. Der Übergang von der Kindheit zur Jugend ist damit durch eine verstärkte Tätigkeit des Einübens charakterisiert. Dennoch ist das Einüben ein Prozess, der sich lebenslang vollzieht, jedoch in unterschiedlichen Quantitäten und Qualitäten. Körperstile verfestigen sich in der Materie des Körpers, die Veränderungs- und Alterungssprozessen ausgesetzt ist. Im Körper wird die Lebensgeschichte des Einübens von Geschlecht und Begehren in dessen Stilen gespeichert, so dass die Verkörperung auf lange Sicht nicht umkehrbar ist, aber dennoch bis zu einem gewissen Grade veränderlich bleibt. 


\section{Literatur}

Bilden, Helga (1991): Geschlechtsspezifische Sozialisation. In: Hurrelmann, Klaus; Ulich, Dieter (Hrsg.): Handbuch der Sozialisationsforschung. Weinheim/Basel, S.279-301

Bilden, Helga/Dausien, Bettina (Hrsg.) (2006): Sozialisation und Geschlecht. Opladen

Bourdieu, Pierre (1997): Die männliche Herrschaft. In: Dölling, Irene/Krais, Beate (Hrsg.): Ein alltägliches Spiel. Geschlechterkonstruktion in der sozialen Praxis. Frankfurt a. M., S. 153-217

Bourdieu, Pierre (1999, Orig. 1980): Sozialer Sinn. Kritik der theoretischen Vernunft. Frankfurt a. M.

Breidenstein, Georg/Kelle, Helga (1998): Geschlechteralltag in der Schulklasse. Ethnographische Studien zur Gleichaltrigenkultur. Weinheim

Breitenbach, Eva (2000): Mädchenfreundschaften in der Adoleszenz. Eine fallrekonstruktive Untersuchung von Gleichaltrigengruppen. Opladen

Breitenbach, Eva/Kausträter, Sabine (1999): „Einarbeiten in heterosexuelle Umgangsformen«: Zur Bedeutung von Mädchenfreundschaften in der Adoleszenz. In: Horstkemper, Marianne/Kraul, Margret (Hrsg.): Koedukation. Erbe und Chancen. Weinheim, S. 184-199

Bruhm, Steven/Hurley, Natascha (Eds.) (2004): Curioser. On the Queerness of Children. Minneapolis

Budde, Jürgen (2005): Männlichkeit und gymnasialer Alltag. Doing Gender im heutigen Bildungssystem. Bielefeld

Bütow, Birgit (2006): Mädchen in Cliquen. Sozialräumliche Konstruktionsprozesse von Geschlecht in der weiblichen Adoleszenz. Weinheim/München

Butler, Judith (1991): Das Unbehagen der Geschlechter. Frankfurt a. M.

Dies. (1995): Körper von Gewicht. Die diskursiven Grenzen des Geschlechts. Berlin

Dies.: Jemandem gerecht werden. Geschlechtsangleichung und Allegorien der Transsexualität. In: Das Argument 242, S. 671-684

Dausien, Bettina/Kelle, Helga (2001): Rekonstruktive Geschlechterforschung. In: Feministische Studien, 19.Jg., H. 2, S. 4-8

Dietze, Gabriele (2003): Allegorien der Heterosexualität. Intersexualität und Zweigeschlechtlichkeit - eine Herausforderung an die Kategorie Gender. In: Die Philosophin. Forum für feministische Theorie und Philosophie, S. 9-35

Faulstich-Wieland, Hannelore/Weber, Martina/Willems, Katharina (2004): Doing Gender im heutigen Schulalltag. Empirische Studien zur sozialen Konstruktion von Geschlecht in schulischen Interaktionen. Weinheim/München

Fritzsche, Bettina (2003): Popfans. Studie einer Mädchenkultur. Opladen

Foucault, Michel (1998): Über Hermaphrodismus. Der Fall Barbin. Frankfurt a.M.

Gebauer, Gunter/Wulf, Christoph (1998): Spiel - Ritual - Geste. Mimetisches Handeln in der sozialen Welt. Reinbek bei Hamburg

Gildemeister, Regine (2000): Geschlechterforschung (gender studies). In: Flick, Uwe/von Kardorff, Ernst/Steinke, Ines: Qualitative Sozialforschung. Ein Handbuch. Reinbek bei Hamburg, S. 213-223

Girard, René (1999, Orig. 1961): Figuren des Begehrens. Das Selbst und der Andere in der fiktionalen Realität. Münster/Hamburg/London

Hackmann, Kristina (2003): Adoleszenz, Geschlecht und sexuelle Orientierung. Eine empirische Studie mit Schülerinnen. Opladen

Hagemann-White, Carol (1984): Sozialisation:Weiblich - männlich? Opladen

Dies.: Die Konstrukteure des Geschlechts auf frischer Tat ertappen. Methodische Konsequenzen einer theoretischen Einsicht. In: Feministische Studien, 11.Jg., H. 2, 9. 68-78

Hartmann, Jutta (Hrsg.) (2004): Grenzvenvischungen. Vielfältige Lebensweisen im Gender-, Sexualitätsund Generationendiskurs. Innsbruck

Hartmann, Jutta/Klesse, Christian/Wagenknecht, Peter/Fritzsche, Bettina/Hackmann, Kristina 
(Hrsg.) (2007): Heteronormativität. Empirische Studien zu Geschlecht, Sexualität und Macht. Wiesbaden

Hengst, Heinz/Kelle, Helga (Hg.) (2003): Kinder - Körper - Identitäten. Theoretische und empirische Annäherungen an kulturelle Praxis und sozialen Wandel. Weinheim/München

Hirschauer, Stefan (1993a): Die soziale Konstruktion der Transsexualität. Frankfurt a. M.

Ders. (1993b): Dekonstruktion und Rekonstruktion. Plädoyer für die Erforschung des Bekannten. In: Feministische Studien, 11.Jg., H. 2, S. 55-67

Honig, Michael-Sebastian/Lange, Andreas/Leu, Rudolf (Hrsg.): Aus der Perspektive von Kindern? Zur Methodologie der Kindheitsforschung. Weinheim/München 1999

James, Allison/Jenks, Chris/Prout, Alan (1998): Theorizing Childhood. Cambridge/Oxford

Jösting, Sabine (2005): Jungenfreundschaften. Zur Konstruktion von Männlichkeit in der Adoleszenz. Wiesbaden

Kelle, Helga/Breidenstein, Georg (1996): Kinder als Akteure. Ethnographische Ansätze in der Kindheitsforschung. In: Zeitschrift für Soziologie der Erziehung und Sozialisation, 16. Jg., H. 1, S. 47-67

Kelle, Helga (2001): "Ich bin der die das macht" Oder: Über die Schwierigkeit, "doing gender"-Prozesse zu erforschen. In: Feministische Studien, Jg. 19, H.2, S. 39-56

King, Vera (2002): Die Entstehung des Neuen in der Adoleszenz. Individuation, Generativität und Geschlecht in modernisierten Gesellschaften. Opladen

Klöppel, Ulrike (2002): XXoXY ungelöst. Störungsszenarien in der Dramaturgie der zweigeschlechtlichen Ordnung. In: polymorph (Hrsg.): (K)ein Geschlecht oder viele? Transgender in politischer Perspektive. Berlin, S. 153-180

Kolip, Petra (1997): Geschlecht und Gesundheit im Jugendalter. Die Konstruktion von Geschlechtlichkeit in somatischen Kulturen. Opladen

Landweer, Hilge: Kritik und Verteidigung der Kategorie Geschlecht. Wahrnehmungs- und symboltheoretische Überlegungen zur sex/gender-Unterscheidung. In: Feministische Studien, 11.Jg., H. 2, S. 34-43

Landweer, Hilge/Rumpf, Mechthild (1993): Kritik der Kategorie "Geschlecht». Streit um Begriffe, Streit um Orientierungen, Streit der Generationen? In: Feministische Studien, 11.Jg., H. 2, S. 3-9

Lang, Claudia (2006): Intersexualität. Menschen zwischen den Geschlechtern. Frankfurt/New York

Lindemann, Gesa (1993): Das paradoxe Geschlecht. Transsexualität im Spannungsfeld von Körper, Leib und Gefühl. Frankfurt a. M.

Maihofer,Andrea (2002): Geschlecht und Sozialisation. Eine Problemskizze. In: Enwägen Wissen Ethik Jg.13. H. 1, S. 13-26

Neue Gesellschaft für Bildende Kunst (Hrsg.) (2005): 1-0-1 [one'o ne] intersex. Zwei-Geschlechter-System als Menschenrechtsverletzung. Berlin

Rottnek, Matthew (1999): Sissies and Tomboys. Gender Nonconformity and Homosexual Childhood. New York and London

Sedgwick, Eve Kosofsky (1985): Between Men. Homosocial Desire in English Literature. New York

Tervooren, Anja (2004): Geschlecht und Begehren in der ausgehenden Kindheit - queere Perspektiven. In: Jutta Hartmann: Grenzverwischungen.Vielfältige Lebensweisen im Gender-, Sexualitäts- und Generationendiskurs. Innsbruck, S. 69-82

Dies.: (2006): Im Spielraum von Geschlecht und Begehren. Ethnographie der ausgehenden Kindheit. Weinheim/München

Winter, Rainer (2001): Die Kunst des Eigensinns. Cultural Studies als Kritik der Macht. Weilerswist

Wulf, Christoph/Althans, Birgit/Audehm, Kathrin/Bausch, Constanze/Göhlich, Michael/Sting, Stephan/Tervooren, Anja/Wagner-Willi, Monika/Zirfas, Jörg (2001): Das Soziale als Ritual. Zur performativen Bildung von Gemeinschaften. Opladen

Wulf, Christoph/Zirfas, Jörg (Hrsg.) (2004): Die Kultur des Rituals. Inszenierungen. Praktiken. Symbole. München 